\title{
Operations Research as an Appraisal of Organization Performance
}

\section{Talatu Muhammad Barwa}

Department of Business Administration and Entrepreneurship, Faculty of Social and Management Science, Bayero University, Kano, Nigeria

\section{Email address:}

talatubarwa05@gmail.com

\section{To cite this article:}

Talatu Muhammad Barwa. Operations Research as an Appraisal of Organization Performance. Science Journal of Business and Management. Vol. 3, No. 4, 2015, pp. 127-133. doi: 10.11648/j.sjbm.20150304.15

\begin{abstract}
The purpose of this study is to carry out research to analyze how operations research can help in increasing the efficiency of the operations performed by the organization. The research is to be carried out through the secondary data available in the context of the topic at hand. The paper focuses on the concept of operations research, the importance of operational research and a detailed description of different business implications of the operations research. Along with the theoretical detail the paper also quotes some examples of the companies that have successfully achieved the organizational efficiency by the implementing the operations research concepts.
\end{abstract}

Keywords: Operations Research, Operation Management, Inventory Management, Inventory System, Organization

\section{Introduction}

Operations research can be defined as techniques of advanced analysis that are used to improve the decision making process of the company. Operations management or the operations research is gaining popularity every passing day because the companies have realized the importance of making the best use of available resources. For organizations to survive in this competitive world, organizations need to look for ways through which they can provide products and services as efficiently and as cost effectively as possible.

\section{Thesis Statement}

The thesis statement for the research paper, state on which the entire research will be carried out is, "Implementation of operational research, in the business operations which leads to an increase in the organizational efficiency".

\section{Mainbody}

Operations research started to be used as a field in the late 1930 's or early 10940's. Operations research is a managerial tool that is used by organizations, no matter what they are either a profit or non-profit oriented organizations. Because the operation research mainly looks for ways through which organization can be made cost effective and improvement can be brought to the operation of the organization (Nenni, 2013). The techniques of operational research are used, in order to help managers make decisions that are economically feasible. With the help of different techniques and methodologies used by the operations research provide the managers with alternatives to decide on. Verifying and validating the available alternatives is a very important component of the decision-making process, most of the operations researches require an intense use of numerical data available to the organization and the analysis of quantitative studies (Nenni, 2013).

Operations research can be used and applied in a number of different fields; one of the main purposes of its use is to resolve the conflicts of interest that can arise while making a strategic decision for the organization (Huisman, 2013). Because if any company chooses to follow a specific way of doing business, there are chances that the conflict of interests between the different departments may arise, and if these conflicts are not resolved they may lead to problems that can hinder the efficiency of the organizations (Nenni, 2013). The need for operations research has been more emphasized by the increase competition, which is a result of the growing global markets.

\section{Operations Research Process}

The operations research procedure is a problem solving process that can be used in order to achieve operational 
efficiencies; it is a process, which comprises of six steps:

\subsection{Analysis of the Problem}

The first step of the process is analysis of the problem that is before looking for the solution we first need to analyze the problem we need solutions for. This analysis gives a basis for the development of the requirements that are to be expected from the model.

\subsection{Construction of the Model}

On the basis of the analyzed problem, the model is constructed. The main purpose for the construction of the model is to look for different ways or techniques that can be used for the solution of the analyzed problem.

\subsection{Deriving the Solution from the Model}

This stage of the process basically helps the analysts to look for the common ground between the analyzed problem and the selected model. The main purpose of this stage is to ensure that all the requirements that were explored during the analysis of the problem stage have been catered properly and accordingly in the selected model.

\subsection{Model Testing}

At this stage, the testing of the applied model is done, that is the analyst or the managers apply the model to the problem or the situation and then analyze the results of the testing. On the basis of the result of the testing phase, the further strategy is created.

\subsection{Analysis of the Testing}

After the testing of the model is done, the results of the testing are then analyzed. If the results show all the positive signs, and it fulfills all the requirements that were expected from the model, then the strategy is formulated to implement the model permanently. In the model implementation strategy an important factor is of the model control, the model will be implemented to the entire system so in order to minimize the chances of any loopholes a control strategy for the model is developed. And in case if the results of the testing phase show that there might be some changes required in the model to achieve the required results, then the improvement of the model will be done to solve all the raised concerns.

\subsection{Implementing the Model}

After the analysis of the model and the up gradation and improvement of the model is done, the model is then implemented in the organization, and the benefits from the model are reaped.

\section{The Significance of Operations Research}

The operations research has a very varied character and can be implemented in a number of different fields. If the researchers involved in bringing innovations in the operations research are able to help the companies to fulfill their required needs, it can be safely said that the field of operations research has a very strong scope (Huisman, 2013). The innovations in the operations management can be also help the companies to play their part in the social improvements of the society. The concept of operations research can be very helpful in bringing a solution to the conflicts that may arise while balancing the conflicts of objectives that are the interest and goals of the organization. The main usage of the concept comes in when there are a number of alternatives available for decision makers, and management is indecisive about which decision to follow (Huisman, 2013). When the concepts of operations research are being followed by an organization, it becomes easier for the organization to resolve the conflicts of interests that may arise between different departments of the organizations.

The operations research aids the concerned departments with suggestions for decision making rules which are based on the following factors: a complete orientation of the system, approaching the problems by scientific methods and the models which are based on quantitative measurements and techniques (Nenni, 2013). Another important point that highlights the importance of operational research is that, it does not only have a use in industrial matters but the techniques used in this concept can be mold and applied for the solution of a number of socio economic problems (Herrmann, 2012).

Different social and societal problems of traffic rules, decision for creating a suitable and reasonable fare structure of public transport, and redefinition of a number of other industrial processes that can bring revolution in the lives of the common people (Huisman, 2013). The use of operations research has also increased in the field of academics and has been used to bring innovations in various aspect of the field. Thus it can be safely said that operations research is a very important field that can help in achieving the efficiency and effectiveness in almost all the fields of life.

\section{Business Applications of Operations Research}

Following are the different approaches or business applications of the concepts of operations research; companies can achieve the organizational efficiencies by following all or few (depending upon the requirements) of these approaches. Each single approach has been explained in a very detailed manner.

\section{Just in Time}

Just in time, generally known as the JIT system, is a strategy used in the production management of the operations research of any company. Just in time is basically an inventory management system in which the 
raw materials required for the production are acquired only when there is a demand for production (Grzechca, 2013). In the $21^{\text {st }}$ century this approach of operations management has gained popularity as this approach enables buyers and sellers as this collaboration between both the suppliers and the manufacturers helps in keeping the inventory costs in control (Grzechca, 2013). This approach enables the manufacturers to produce goods and services on the demands of consumers while keeping the costs in control. The purpose of using the just in time approach is to help the businesses from investing high amounts on inventory storage systems, especially in cases when inventory is more than what is required for production. This surplus inventory requires special storage facilities that can make the inventory cost go very high (Alippi, Boracchi \& Roveri, 2013).

\subsection{Just in Time from the Manufacturers Side}

From the perspective of a manufacturing concern just in time can be beneficial in many ways, but there are two most important aspects of the system that can help the company to make most of it. The first is that if the company follows just in time system, it will not need to make extra investments on the storage facilities. Because, by following the just in time approach of operations research application, the company will not need to make efforts for the storage of the extra inventory of raw materials but rather it will order raw materials and make the manufacturing without the need of storing the materials (Alippi, Boracchi \& Roveri, 2013).

Secondly, when the product is ready even then it will not need to store the inventory but instead it can manufacture the product and provide it to the end product deliverers. Also another important point to note in just in time approach is that this approach follows a demand pull strategy so the production is only done when there is a demand for the product on the other side. Also when a company is not following a just in time approach, and it is producing the products on the basis of its market assumptions there are very high chances that the business might end up with excessively produced goods. These undemanded goods might become a risk of loss for the company (Alippi, Boracchi \& Roveri, 2013).

\subsection{Just in Time from the Retailers Side}

The same approach when used by the retailers can also be beneficial for them. Retailers, in order to save themselves from the potential loss that can be caused if the product goes out of stock, always prefer having some extra inventory in the stock. Here the retailers need to take help from their analysis of market sales projections because the retailer should not keep inventory in such a short number that a slight increase in demand causes a shortage of product. Similarly, a product should also not be in such an excess quantity that if the demand for the product does not increase, it directly affects the business and the business faces loss (Grzechca, 2013).

\subsection{The Risks Involved in Just in Time System}

The just in time, inventory management system is generally regarded as one of the most efficient ways of operations research application used for managing of the inventory system. However, along with huge lists of benefits there are some major risks involved if any business follows the just in time inventory management system. In order for the just in time system to work properly the supply chain system of the company has to be extremely strong and error proof, because a small malfunction of the supply chain can lead to huge raw material or stock shortages for companies who follow this system. This risk becomes extremely high when the raw materials used for manufacturing are needed to be imported from any other place. However, importing the raw material is not the only exceptional case, even if the raw materials are being purchased locally, if there is a small glitch in transportation or the delay in the shipment delivery of the raw materials can cause the inventory go out of stock; thus making the manufacturing of the product problematic (Alippi, Boracchi \& Roveri, 2013).

Just in time systems are successfully being used by a number of organizations in the world, the main philosophy behind the just in time approach is to eliminate the wastes and reduce the cost that is incurred by a company on the production of goods. The main objective behind the usage of this approach is to help the company to produce the right product, in the right quantity, at the right place on the right time. In the just in time system, very little or no finished product is kept in stock and no excess products are produced that can be kept in stock.

\section{Total Quality Management}

The total quality management which is generally also known, as TQM is a system of operations research approach that is used by companies in order to achieve success. The approach suggests that all the members of an organization are put in equal efforts and participate in the improvement of the products, services and the process through which the processes of the organization (Asif, Vries \& Ahmad, 2013). Generally, the total quality management is followed by organizations that are customer driven; the approach requires a continual improvement from the side of employees. Various strategies, effective communications and data are used to integrate such systems in the organizations which are quality driven; all the cultures and the activities of the organization are highly dependent on quality factors (Chan, Neailey \& Ip, 1998). The total quality management helps the organization to improve the quality of the products and services that are being produced by the company. Many times these improvements are done on the basis of the feedback, which is usually taken from the consumers (Iyer, Saranga \& Seshadri, 2013).

There are some elements of total quality management, these are as under:

In the total quality management all the employees of the 
company work together in the achievement of a common goal, the goal of achieving the best quality for manufactured goods and services. Employee commitment, employee empowerment and the provision of the right environment for the employees are one of the important factors for the achievement of the total quality management in the operations of a company (Chan, Neailey \& Ip, 1998). And also when the "high performance work systems" are integrated in the organization these are also efforts taken by the organization in order to achieve efficiency in the operations (Asif, Vries \& Ahmad, 2013).

Process thinking or also known as the process innovation is also a very fundamental part of the total quality management. The processes of every organization are different from those of the other organizations, however in general a process may be defined as the series of the steps taken from the inputs of suppliers and transform them after the processing into something (that is the output) that can be delivered to the end consumer. All these steps that are needed for the fulfillment of the requirements of the production are predefined and the performance of the employees is then continuously monitored (Asif, Vries \& Ahmad, 2013).

The most important aspect of the total quality management is to bring integration to all the processes of the organization. In every organization there are a number of processes involved for the achievement of the core business activities of a company, and for any organization to achieve the successful completion of its business functions a harmony among these activities is very important (Martin, 2013). The main purpose total quality management is to provide integration to the activities that are horizontally connected, however, the vertically connected activities are also not ignored. According to the concepts of total quality management all the small level processes of the company are very important in formulating the macro level strategies of the company (Iyer, Saranga \& Seshadri, 2013).

There should be a clear description of the vision \& mission of the company and also the policies and the objectives of the company. After the description of the business processes then it becomes relatively easier for the quality assurance department of the company to monitor the performance of all the business units, after monitoring the result of this monitoring should be communicated to the employees of the organization as well (Martin, 2013).

Along with the quality assurance of all the other factors another very important aspect is of the quality assurance of the work and environmental culture of the organization, every organization has a very unique set of culture, this culture is not only the distinctive factor of the organization, but it is also something that helps an organization with the achievement of operational excellence (Martin, 2013).

In order to ensure the successful achievement of quality assurance, it important to realize that the performance of the organization is measured against the set policies. For this purpose, the total quality management requires an organization to collect and analyze data on the continual basis so that the accuracy in the future decision-making can be achieved. Along with the above stated factor it is also very important that all the important aspects as well as the decisions of the company are properly communicated to the respective employees. Because the best policies if not rightly communicated may become an unfruitful process for the company (Iyer, Saranga \& Seshadri, 2013).

All the factors that are stated above are the ones that are measured as a very important part of the application of total quality management in the organization; these factors should be treated and communicated as the core values or defining the principles of the organization. And the successful application of total quality management in the organization will help the organization to achieve success in all the aspects of its business operations (Asif, Vries \& Ahmad, 2013).

\section{Six Sigma}

Six Sigma is a concept of operations research that can be defined as a set of tools and techniques that can be used for the improvement of the different processes that are being followed in a company. A very famous telecommunication company, Motorola, developed the concept of Six Sigma in the year 1986. Today, the concept of Six Sigma is highly used by a number of companies, and these companies are achieving unmatched success with the application of this approach (Ismyrlis \& Moschidis, 2013). Six Sigma can be called an operations management philosophy, which puts emphasis on setting high objectives, collection of data and the analysis of the results in such a way that the defects in the products and services that are being produced by the company are reduced to the lowest possible levels. Apart from all its different definitions, the concept of Six Sigma now has an open range of interpretations; these interpretations are beyond the control and reach of the initial originators of the concept. Six Sigma is a very famous and vastly used method for initiation of the organizational change and to support the process of this change. Six Sigma is a very flexible concept; it might be "production quality metric" for an engineer but for the rest of the organization and the stake holders of the company, who do not have a direct link to the implementation of this process, Six Sigma might be a corporate culture (Ismyrlis \& Moschidis, 2013).

Six Sigma helps in the improvement of the quality of the processes of the manufactured products by identification and removal of the causes of the errors and defects in the manufacturing processes. Six Sigma can be called a measure of quality, which attempts to achieve results near perfection. The description of defects in the concept of Six Sigma is said to be of something that is outside the specifications provided by the customer. The main purpose of the Six Sigma is to implement the strategies which are measurement based and focus on the improvement of the process and the reduction of the chances of variation that can occur while the operations are being performed (Ismyrlis \& Moschidis, 2013). 


\subsection{The Six Sigma Process}

When an organization decides to implement the Six Sigma approach in the company, the executive level management first needs to decide upon the strategies that can be followed for the fulfillment of the improvement initiatives. The focus of these strategies however, should be on improvement of the processes that are essential to meet the requirements of the customers. The team of managers who own the responsibility of the implementation of these processes has to perform the following tasks: to understand and identify the processes in detail, then to understand the level of quality that is expected by the customers of the product. After the understanding of the requirements, which are expected out of the system, the measurement of the effectiveness and the efficiency of each process performance is done, that is to measure "the number of defects per million operations".

The theory of Six Sigma is entirely logical to understand that is when all the important delivery chain processes are properly understood and the loopholes in the system are removed. These actions will have a direct impact on the operational processes of the company and there will be increased efficiency achieved by the organization, this increased efficiency will lead the company to achieve competitive advantage, this competitive advantage of the company will help it to achieve the increased customer satisfaction and eventually increasing the profitability of the company (Ismyrlis \& Moschidis, 2013).

The implementation of the Six Sigma concepts is not an easy job to do; therefore Six Sigma requires the managers and the team leaders to take complete responsibility for implementing the Six Sigma strategies. When a company follows the Six Sigma approaches it needs to properly train all its employees about the applications of the Six Sigma methodologies.

The trainings of the employees include: how to use the improvement and the measurement tools and also to fulfill the needs of the internal and the external stakeholders for the company, that include suppliers and the end consumers, trainings for the improvement of communications and relationship skills is also done. Because the concepts and the applications of the methodologies of the Six Sigma are very difficult to understand and even more difficult to implement, the proper trainings of the employees are very crucial part of the Six Sigma implementation (Ismyrlis \& Moschidis, 2013).

\subsection{Example}

From the list of the companies that have implemented Six Sigma successfully and have benefited from the application of the approach in their operations activities, General Electric is one of the first few companies that followed the footsteps of Motorola. GE has successfully implemented the Six Sigma and has been reaping the benefits for almost two decades now. Realizing the benefits that can be achieved thousands of companies have implemented the concepts of Six Sigma in their business operations and through the implementation of Six Sigma the companies have earned millions and millions of profits. The General Electronics and the Motorola, who were the initiator of these processes, developed a certification program, under which both the companies started giving trainings about the methodologies and the implications of the concept. The individuals who attended the training sessions were then given the verification certifications and they were called as the Six Sigma experts, after the popularity of the concepts, many companies followed the footsteps of the initiators and started conducting the training sessions for their employees and gave them certifications on the successful completion of the training.

\section{World Class Manufacturing System}

World class manufacturing system can be defined as a tool that is used by companies to be enabled to perform at a best world-class level; the world class manufacturing system was first adopted by the very famous automobile company Fiat. Companies all around the world face intense competitiveness, these companies, in order to achieve an edge over their competitors must produce goods and services of very high quality. But production of high quality goods and services cannot become a sole factor for any company's profitability because if the production of these goods is left without a cost check element, there will be no profitability for the company. So when it comes to producing goods and services of high quality, ensuring that the high quality is achieved by keeping the cost in control can lead the company to achieve higher levels of profitability.

The companies that follow the world class manufacturing systems, they do so by integrating all systems of manufacturing in such a manner that the needs of the customers are met very effectively and efficiently. Here another important factor to notice is that the companies that are striving to become world class manufacturers they should place very high importance to the technological improvement, usage of up to dated equipment and even the manufacturing processes of the company should also be very innovative with constant improvements as and when needed.

\subsection{Industrial Engineering}

Industrial engineering is used to achieve the improvement in the production systems of a company; industrial engineering is a factor that can be used to bring success in the companies that are involved in the process of mass production. The concept of industrial engineering became the basis for the management consultancy and the business administration policies that are prevailing in the world today. Any companies that want to achieve the organizational efficiencies can also say industrial engineering as a technology that combines both the product technologies and product specific manufacturing techniques followed.

\subsection{Example}

The implication of operations research applies the 
statistical analysis and the usage of different mathematical models to solve a number of business problems that are faced by different organizations and also help the companies to come up with improved decision-making processes. As the complexities of the businesses' environment increases, the companies and governmental departments rely more and more on the analyses that are based on the management intuition. Implementation of operations research in the organization has helped a number of companies achieve increased operational efficiency. Let's take an example of $\mathrm{P} \& \mathrm{G}$, which has an annual sale of more than 76 billion dollars and operations in more than 80 countries of the world The operations of worlds this largest consumer good company is so huge that it is said to be an economy unto itself. In recent years the company has become very active in data innovation and the steps taken by the company in the field of operations research has become the competitive edge of $P \& G$ and has brought enormous success to the company. With the help of operations research $P \& G$ has achieved a number of operational efficiencies. The company streamlined its manufacturing processing plants; used new product branding strategies for its various brands, operations research helped $P \& G$ to streamline it's all the sourcing of raw materials. Using the different business applications of operations research $\mathrm{P} \& \mathrm{G}$ achieved efficient inventory management systems and redefined the entire organizational design.

Another example of the company that has achieved enormous operational efficiencies by the implication of operations research is the giant Coca Cola Enterprise, the company is among one of the world's top brands. Coca cola enterprise with the help of operations research concepts streamlined a number of its supply chain issues and achieved operational efficiencies.

Samsung is the largest Smartphone seller company in the world; the operations of this giant of its industry are very diverse but the company has managed to not only been able to streamline its production processes, but it has also achieved promise filling supply chain systems. And the company was able to do that by making the right decisions of focusing on the operations research models, and apply them according to the needs of the company. Samsung has mainly achieved the growth through its unmatched international sales and in order to be successful in that domain the company had to maintain most innovative supply chain and logistics services. The company achieved them by implementing the operations research approaches in its operations.

Ford Motors, which is a strong player of the automobile industry, has also achieved organizational efficiency by operations research. The company is the inventor of the concept of the assembly line; the idea revolutionized the entire system of manufacturing in the automobile industry. From the very beginning this company has always put a very high focus on looking for the innovative ways of revolutionizing the operations management. Ford has been able to achieve the success in bringing innovation in its operations by focusing on the operations research.
DHL, which does business in the industry of "international Express and Logistics industry", the company, is the market leader of its industry. Being a logistics company, the success of DHL has always revolved around providing its customers with the unmatched services at the fastest possible speed. By putting a focus on the operations research DHL redefined the strategic vision of the company by brining an alignment in both its internal support systems and the buying behaviors of its customers. The company has smartly made use of reverse engineering and aligned its operations with customer preferences. After the company successfully implemented the strategies it carried out a survey to measure the effect of the operations research, the survey showed that there was an increase in the market share of the company, the company also enjoys a very high customer satisfaction.

The other example of a company that has achieved organizational efficiency by Boeing, Boeing which is one of the world's greatest manufacturers of heavy machinery has achieved this huge success by implementing the operations research approaches in their manufacturing processes this has enabled the company to achieve the organizational efficiencies.

\section{Conclusion}

The different methods, through which the operations research can be achieved, should be used according to the need of the operations required by the organization. Different methods can be used for different purposes, but what needed is, that there should be an alignment in the systems that are being used. The managers are also the decision makers for any organization; the overall performance of any organization is highly dependent on the operational performance of the company, and the operational managers play a very imperative role in implementing the operations research approaches with the core businesses operations of the company.

\section{References}

[1] Alippi, C., Boracchi, G., \& Roveri, M., (2013), "Just-In-Time Classifiers for Recurrent Concepts", IEEE Transactions on Neural Networks and Learning Systems, pp. 620-634.

[2] Aoun, M., \& Hasnan, N., (2013), "Lean production and TQM: Complementary or Contradictory Driving Forces of Innovation Performance?", International Journal of Innovation Science, pp. 237-252.

[3] Asif, M., Vries, H., \& Ahmad, N., (2013), "Knowledge creation through quality management", Total Quality Management \& Business Excellence, pp. 664-677.

[4] Chan, Y., Neailey, K., \& Ip, W., (1998), "ISO 9004-2 Quality Management System - The Way to World-Class Service”, Managing Service Quality, pp. 395-401.

[5] Dyson, R. (2000), "Strategy, performance and operational research", Journal of the Operational Research Society, pp. 511. 
[6] Grzechca, W. (2013), "Just in Time Strategy in Balancing of Two-Sided Assembly Line Structure", International Journal of Materials, Mechanics and Manufacturing, pp. 65-70.

[7] Herrmann, J., (2012), "Handbook of operations research for homeland security", New York: Springer, pp. 45-50.

[8] Huisman, D., (2013), “Operations research proceedings 2013: Selected papers of the International Conference on Operations Research", OR2013, organized by the German Operations Research Society (GOR), the Dutch Society of Operations Research (NGB) and Erasmus University Rotterda, n.p.

[9] Ismyrlis, V., \& Moschidis, O., (2013), "Six Sigma's critical success factors and toolbox", International Journal of Lean Six Sigma, pp. 108-117.

[10] Iyer, A., Saranga, H., \& Seshadri, S., (2013), "Effect of Quality Management Systems and Total Quality Management on Productivity Before and After", Production and Operations Management, pp. 283-301.

[11] Martin, M. (2013), "Business efficiency for dummies", John Wiley \& Sons Inc., pp. 55-60.

[12] Nenni, M., (2013), "A Cost Model for Integrated Logistic Support Activities", Advances in Operations Research, pp. 1-6.

[13] Pišáková, M., \& Kozlovská, M. (2013), "Lean Production as an Innovative Approach to Construction", Selected Scientific Papers - Journal of Civil Engineering, n.p.

[14] Saurin, T., Rooke, J., \& Koskela, L., (2013), “A complex systems theory perspective of lean production", International Journal of Production Research, pp. 5824-5838.

[15] Sugumaran, V., (2013), "Organizational efficiency through intelligent information technologies", Information Science Reference, n.p. 